\title{
Clinical referral patterns and cognitive profile in mild cognitive impairment
}

\author{
Jane A. Lonie, Lucie L. Herrmann, Claire L. Donaghey and Klaus P. Ebmeier
}

\section{Background}

There is current interest in exploring the different subtypes of mild cognitive impairment $(\mathrm{MCl})$, in terms of both their epidemiology and their cognitive profile.

\section{Aims}

To examine the frequency of $\mathrm{MCl}$ subtypes presenting to a memory clinic and to document detailed neuropsychological profiles of patients with the amnestic subtype.

\section{Method}

Consecutive tertiary referrals $(n=187)$ were psychiatrically evaluated; 45 patients met criteria for amnestic mild cognitive impairment (aMCl). A subgroup of 33 patients with $\mathrm{aMCl}$ as well as 21 healthy controls took part in a thorough neuropsychological examination.

\section{Results}

Of the patients who were examined in greater neuropsychological detail, ten had pure $\mathrm{aMCl}$ (none with visual memory impairment only). Fifteen met criteria for nonamnestic MCl. Fifteen had normal neuropsychological profiles. Using more than one test increased sensitivity to detect episodic memory impairment.

\section{Conclusions}

Amnestic $\mathrm{MCl}$ is an important diagnosis in secondary and tertiary memory clinics. There is scope to improve the efficacy and sensitivity of the clinical assessment of this impairment.

\section{Declaration of interest}

None.
The diagnosis of mild cognitive impairment (MCI) represents an attempt to define features of the dementias in their preclinical phases. Petersen and colleagues developed research criteria with a cut-off for verbal recall performance as objective evidence of episodic memory impairment. ${ }^{1}$ This approach has been challenged because it excludes patients who display exclusively visual episodic memory impairment. ${ }^{2}$ Moreover, the pure amnestic subtype of mild cognitive impairment is rare,$^{2-6}$ and mild cognitive impairment case definition varies as a function of the neuropsychological tests used. ${ }^{2,7}$ It is not clear how the Petersen criteria might best be translated into clinical practice. There is little information detailing the frequency with which each of the subtypes presents to memory clinics. Our aim was to examine the diagnostic profile of patients with amnestic mild cognitive impairment (aMCI) referred to our tertiary assessment service. We also sought to evaluate a comprehensive battery of neuropsychological measures for their usefulness in this patient group.

\section{Method}

This study constituted part of a longitudinal project for which ethical approval was obtained from the local research ethics committee. In accordance with this, informed consent was given by all participants.

\section{Sample}

We retrospectively analysed 187 consecutive referrals to the Edinburgh Neuropsychological Assessment Service for Older People between the months of September 2004 and April 2006. Referrals were received at a tertiary level, stemming from consultants in old age psychiatry, geriatric medicine and neurology. All of these patients had undergone comprehensive psychiatric evaluation, relevant medical screening (including a standard battery of screening blood tests) and neuroimaging (computed tomography and/or magnetic resonance imaging or single-proton emission computed tomography) prior to being referred to our service. All but three patients were over the age of 50 years.

The original criteria for mild cognitive impairment set out by Petersen et al require that a person must present with a memory complaint, show evidence of objective memory decline in relation to age and education, demonstrate preservation of other areas of cognitive function and activities of daily life, and not fulfil criteria for dementia. ${ }^{8}$ Because it has since become apparent that not everyone who demonstrates cognitive impairment short of dementia has a 'memory' complaint, we used the recently expanded criteria that include people with non-memory complaints (single-domain non-memory $\mathrm{MCI}$ ), as well as those exhibiting multiple domains of cognitive impairment who none the less fail to fulfil criteria for dementia (multiple domains slightly impaired)..$^{9,10}$ The Mini Mental State Examination $(\mathrm{MMSE})^{11}$ and Addenbrooke's Cognitive Examination ${ }^{12}$ were administered as a means of establishing the participants' general level of cognitive functioning. Level of everyday functioning was examined by means of the Clinical Dementia Rating scale ${ }^{13}$ within the context of a clinical interview with the patient and the patient's primary carer (when available). A total of 112 patients fulfilled one or more of the following exclusion criteria and were therefore excluded from the analyses:

(a) dementia (MMSE score $<24 / 30$ or ACE score $<80 / 100$, plus fulfilling DSM-IV criteria; ${ }^{14}$

(b) depression, assessed either by way of formal psychiatric consultation or, in a small proportion of cases, by a score greater than 10 on the Geriatric Depression Scale ${ }^{15}$ or clinical assessment by one of the authors (J.A.L.);

(c) one or more medical or psychiatric conditions that could conceivably account for the patient's cognitive impairment (head injury, schizophrenia, evidence of stroke or tumour on neuroimaging, alcoholism, epilepsy, cranial radiotherapy).

Of the remaining 75 patients, 15 showed cognitive impairments outside the domain of episodic memory, 15 returned a 'normal' cognitive profile and 45 showed memory function 
impaired for age (with or without additional areas of cognitive impairment). We present detailed neuropsychological baseline findings for 33 of these 45 patients as well as for 21 healthy individuals from the community who agreed to participate as a control group in a continuing longitudinal study examining neuropsychological markers of preclinical dementia. Control group participants were recruited through a local dementia support group or were spouses or carers of patients who had attended the neuropsychological assessment service.

\section{Neuropsychological assessment measures}

All the participants were given a comprehensive battery of neuropsychological tests. These tests were selected on the basis of their demonstrated validity for use within a population with MCI, and assessed the primary domains of verbal and visual episodic memory, semantic memory and language, processing speed, attention/executive function and visuospatial ability.

\section{Premorbid intellectual ability}

The National Adult Reading Test (NART) ${ }^{16}$ was administered in order to assess probable premorbid level of intellectual function.

\section{Episodic memory}

To assess verbal episodic memory, participants were given the Hopkins Verbal Learning Test - Revised (HVLT-R). ${ }^{17}$ In this test participants are asked to recall as many words as possible immediately after presentation of a 12 -item word list on three consecutive learning trials. Measures included total number of words recalled across three registration trials (maximum 36), total number of words recalled following a 30 min delay (maximum 12) and a discrimination index score representing a participant's ability to discriminate between old and new list items. Visual episodic memory was assessed by means of two different tasks. The CANTAB Paired Associate Learning (PAL) test is a computerised measure of visuospatial learning ability requiring participants to learn the locations of an increasing number of patterns - one, two, three, six and then eight; ${ }^{18,19}$ the score of interest was the number of pattern-position errors at the six-pattern level. Participants were also administered the Rey Complex Figure Test. ${ }^{20}$ For this test, participants are asked to make a copy of a complex figure, with no time restriction. Immediately after presenting the figure, and again following a 30 min delay, participants are required to make another copy from memory.

\section{Semantic memory}

Participants completed the Graded Naming Test, ${ }^{21}$ the Graded Faces Test, ${ }^{22}$ the Boston Naming Test ${ }^{23}$ and the Edinburgh Exemplar Naming Test (EENT; further details available from the authors). The Graded Naming Test and Boston Naming Test require participants to name line drawings of increasing difficulty, whereas the Graded Faces Test requires participants to name a series of 30 famous faces. ${ }^{22}$ The EENT was developed by one of the authors (J.A.L.) in an effort to improve the sensitivity of existing confrontation naming measures to early semantic memory failure. In this test the participant is required to name 50 line drawings of low-frequency animate objects with sizeable feature overlap. Participants were also asked to complete a category fluency task, requiring them to name as many animals as they could in $1 \mathrm{~min}$.

\section{Attention/executive functioning}

As a means of examining attention/executive function and visuomotor processing speed, participants were asked to produce as many words as possible beginning with the letter $\mathrm{P}$ in $1 \mathrm{~min}$ (letter fluency task). In addition, participants were administered parts A and $\mathrm{B}$ of the Trail Making Test: ${ }^{24}$ in this test participants are required to join up as quickly as possible numbered circles in ascending order (part A) and numbers and letters in ascending alternating sequence (part B), while the time to completion is recorded.

\section{Visuospatial skills}

Visuospatial skills were assessed by means of the Rey Complex Figure Test copy task described above. ${ }^{20}$

\section{Comparison with other memory clinics}

We searched the literature for studies employing neuropsychological test batteries similar to ours to examine the comparability of our sample with other published data.

\section{Statistical analysis}

We calculated $z$-scores to determine where scores fell below the tenth percentile of control performances. Visual inspection together with a one-sample Kolmogorov-Smirnov goodness-of-fit test indicated that the data were normally distributed. Group means were compared using independent sample $t$-tests. To determine whether there is an association between general level of cognitive function and the consistency of episodic memory impairment, we divided the participants with cognitive impairment into two groups: those who displayed episodic memory impairment on a single measure only and those who showed impairment on two or more episodic memory tests. Their Addenbrooke's Cognitive Examination and MMSE scores were compared using an independent samples $t$-test. Diagnostic categories between memory clinics were compared using chi-squared tests for diagnostic categories.

\section{Results}

\section{Literature search}

We identified one other study reporting consecutive referrals to a memory clinic using similar diagnostic criteria and assessment measures. $^{2}$

\section{Comparability of referral patterns and cognitive profiles}

A striking similarity in referral patterns was observed between our Neuropsychological Assessment Service for Older Adults and data reported recently from the Cambridge Memory Clinic. ${ }^{2}$ When the 150 pre-excluded referrals from the Cambridge Memory Clinic were accounted for, over half $(60 \%)$ of referrals from both centres were excluded on the grounds of an established dementia or depressive disorder, or one or more medical conditions that could account for the patient's cognitive impairment $\left(\chi^{2}=0.015\right.$, $P=0.90)$. Close to $40 \%$ of referrals from both centres fell within the non-demented and non-depressed category $\left(\chi^{2}<0.001\right.$, $P=0.995)$. Just over half of these patients in both centres met Petersen's expanded criteria ${ }^{9}$ for aMCI $\left(\chi^{2}=0.46, P=0.50\right)$, representing close to a fifth of overall referrals from both centres. Of the remaining $40 \%$ of patients in the non-demented, non-depressed category, half demonstrated cognitive deficits of a non-amnestic variety (in one or more domains) and half returned 'normal' cognitive profiles. Although referral patterns for aMCI were similar 
across the two centres, there was a greater proportion of patients with non-amnestic MCI and fewer with visual-only impairment in our sample $\left(\chi^{2}=13.23\right.$, d.f. $=3, P=0.004$; Fisher-Freeman-Halton exact test, $P=0.003)$. Mean scores for both the aMCI and control groups across all neuropsychological measures were similar to those previously reported.

\section{Sample characteristics}

Our final sample for analysis consisted of 33 patients with aMCI (13 men and 20 women, with a mean age of 74.0 years, s.d. $=6.4$; social class I $n=9$, II $n=12$, IIIN $n=9$, social class not known $n=3$ ) and 21 healthy community-dwelling older adults without cognitive complaints ( 7 men and 14 women, with a mean age of 69.5 years, s.d.=7.4). These groups did not differ in terms of estimated premorbid level of intellectual function. The mean age of our control group was, however, significantly lower than that of our aMCI patient group, a finding similar to previous reports. $^{2,4}$

\section{Comparison of aMCl and control groups}

Despite a mean ACE score that exceeded suggested cut-off points for dementia, ${ }^{12}$ the aMCI group had significantly lower mean scores than the control group on all neuropsychological measures, with the exception of the Rey Complex Figure Test copy task, the Trail Making Test part A and the letter (phonemic) fluency task. These findings were confirmed when the analysis was re-run with age as a covariate, with the exception of performance on the CANTAB PAL and the Trail Making Test part B, which just failed to reach significance. Demographic data and mean scores for the two groups on the individual neuropsychological measures are provided in Table 1. All aMCI group means are based on data from 33 participants, with the exception of the final three semantic memory measures, for which the participant numbers ranged between 13 and 31 .

\section{Amnestic $\mathrm{MCl}$ group performance}

\section{Episodic memory}

The neuropsychological performances of 33 of the 45 patients who fulfilled Petersen's expanded criteria ${ }^{9}$ for aMCI were examined in greater detail. As in the Cambridge Memory Clinic study, ${ }^{2}$ not all of these participants demonstrated impairment across all episodic memory measures: 11 (33\%) showed impairment on a single test, $9(27 \%)$ showed impairment on two memory measures and the remaining 13 (39\%) were impaired on three or more tests. Mean MMSE and Addenbrooke's Cognitive Examination scores for participants who were impaired on more than one episodic memory measure were significantly lower than for those showing impairment on a single test $(P<0.05)$. Just over half of our aMCI subgroup showed both verbal and visual episodic memory impairment. Although a significant proportion (45\%) demonstrated memory impairment of a verbal nature only, none of our patients in this group exhibited a pure visual memory deficit.

\section{Non-memory measures}

Only ten $(30 \%)$ patients in our aMCI subgroup exhibited an isolated impairment of episodic memory function. All the other patients $(70 \%)$ exhibited deficits in one or more additional domains of cognition, most commonly that of semantic memory function, followed by attention and executive function (Table 2).

\begin{tabular}{|c|c|c|c|}
\hline & $\begin{array}{l}\text { Control group } \\
\qquad(n=21) \\
\text { Mean (s.d.) }\end{array}$ & $\begin{array}{l}\text { aMCl group } \\
\qquad(n=33) \\
\text { Mean (s.d.) }\end{array}$ & $\begin{array}{l}\text { Control } \\
\text { v. aMCl } \\
P\end{array}$ \\
\hline Age, years & $69.8(7.4)$ & $74.0(6.4)$ & $0.02^{*}$ \\
\hline NART score & $118.3(2.8)$ & $116.4(8.5)$ & 0.331 \\
\hline MMSE score $(30)^{\mathrm{b}}$ & $29.1(0.8)$ & $28.0(1.8)$ & $<0.01^{* *}$ \\
\hline $\begin{array}{l}\text { ACE score } \\
\text { Total (100) } \\
\text { Delay }(7) \\
\end{array}$ & $\begin{array}{r}94.8(3.3) \\
6.4(0.9)\end{array}$ & $\begin{array}{r}88.2(5.9) \\
3.9(2.3)\end{array}$ & $\begin{array}{l}<0.001^{\star \star *} \\
<0.001^{\star \star *}\end{array}$ \\
\hline $\begin{array}{l}\text { Rey Complex Figure Test scores } \\
\text { Copy (36) } \\
\text { Immediate recall (36) } \\
\text { Delayed recall (36) }\end{array}$ & $\begin{array}{l}34.0(2.4) \\
19.1(6.3) \\
17.4(7.4)\end{array}$ & $\begin{array}{l}34.2(2.5) \\
12.3(6.0) \\
10.8(6.9)\end{array}$ & $\begin{aligned} & 0.729 \\
< & 0.001 * * \\
< & 0.01 * *\end{aligned}$ \\
\hline $\begin{array}{l}\text { HVLT-R score } \\
\text { Total recall (12) } \\
\text { Delayed recall (12) } \\
\text { Discrimination (12) } \\
\text { PAL } 6 \text { errors }\end{array}$ & $\begin{array}{l}24.3(5.0) \\
8.19(2.8) \\
10.3(1.9) \\
7.8(6.9)\end{array}$ & $\begin{aligned} 18.2 & (4.6) \\
4.8 & (3.2) \\
8.5 & (2.2) \\
16.6 & (14.8)\end{aligned}$ & $\begin{array}{l}<0.001^{* * *} \\
<0.001^{* *} \\
<0.01^{* *} \\
<0.01^{* *}\end{array}$ \\
\hline $\begin{array}{l}\text { Trail Making Test score } \\
\text { Part A, S } \\
\text { Part B, S }\end{array}$ & $\begin{array}{l}40.3(11.2) \\
87.5(31.6)\end{array}$ & $\begin{array}{r}48.4(20.0) \\
131.7(78.4)\end{array}$ & $\begin{array}{c}0.095 \\
<0.01 * *\end{array}$ \\
\hline Animal fluency score & $21.1(5.7)$ & $15.1(4.5)$ & $<0.001^{\star \star \star}$ \\
\hline P words score & $15.7(5.8)$ & $16.0(4.8)$ & 0.860 \\
\hline Boston Naming Test score (60) & $57.4(3.1)$ & $53.6(5.5)$ & $<0.01 * *$ \\
\hline EENT score (50) & $46.8(3.0)$ & $43.6(4.5)$ & $<0.01^{\star *}$ \\
\hline $\begin{array}{l}\text { Graded Faces Naming Test } \\
\text { score (30) }\end{array}$ & $20.8(3.1)$ & $16.3(4.9)$ & $<0.001^{* \star *}$ \\
\hline Graded Naming Test score (30) & $23.4(3.2)$ & $19.5(4.2)$ & $0.015^{*}$ \\
\hline \multicolumn{4}{|c|}{$\begin{array}{l}\text { ACE, Addenbrooke's Cognitive Examination; aMCI, amnestic mild cognitive } \\
\text { impairment; EENT, Edinburgh Exemplar Naming Test; HVLT-R, Hopkins Verbal } \\
\text { Learning Test - Revised; MMSE, Mini Mental State Examination; NART, National Adult } \\
\text { Reading Test; PAL, CANTAB Paired Associates Learning test. } \\
\text { a. Independent sample } t \text {-tests comparing the two groups. } \\
\text { b. The maximum test score is given in parentheses after each test name in the left-hand } \\
\text { column. } \\
\text { * } P<0.05 ; P<0.01 ; P<0.001 \text {. }\end{array}$} \\
\hline
\end{tabular}

\section{Discussion}

\section{Referral patterns}

In this study we have shown that people who are neither depressed nor demented but who fulfil Petersen's expanded criteria ${ }^{9}$ for aMCI make up a significant proportion of referrals to our Old Age Clinical Neuropsychology service. Roughly a quarter of all patients referred during an 18-month period met Petersen's criteria, of whom a minority exhibited a memory deficit in isolation following comprehensive neuropsychological examination. This is an almost identical proportion of patients to that reported in the study by the Cambridge Memory Clinic (23\%), ${ }^{2}$ and is a very similar figure to the $20 \%$ of patients seeking help in Lehrner et al's clinic who met criteria for aMCI. ${ }^{25}$ Furthermore, the observation that the highest numbers of people with MCI were those with more than one domain of cognitive deficit is in keeping with the findings from both population-based and other memory clinic studies. $^{7,26}$

It is possible that referral patterns might differ depending on whether the memory clinics are geriatrician- or psychiatrist-led. Surprisingly, the level at which referrals are received (whether primary, as in the Cambridge Memory Clinic, or tertiary, as in our clinic) appears not to influence the proportion of referrals of an MCI nature that are received.

Thus, it appears that both the concept and criteria are applicable and, indeed, a necessary adjunct to clinical practice. 
Table 2 Performance of patients with amnestic mild cognitive impairment ( $n=33$ ) on individual episodic memory measures and non-amnestic measures

Patients performing below
10th percentile of control
group performance
$n(\%)$

Episodic memory

HVLT-R total

HVLT-R delay

$17(52)$

HVLT-R discrimination index 13 (39)

Any HVLT-R measure 24 (73)

PAL errors stage 6

Rey test delay 11 (33)

ACE delay $23(70)$

Patients with impairment 11 (33)

on 1 measure only

Patients with impairments 9 (27)

on 2 measures

Patients with impairments

on 3 measures

Patients with impairments 7 (21)

on 4 measures

Semantic memory/language

BNT 13 (39)

EENT $11(33)$

GFT $12(36)$

GNT 4 (12)

Animal fluency $17(52)$

Participants showing impairment on 22 (67)

one or more semantic memory measure

Attentional/executive function

$P$ words 1 (3)

TMT part B 11 (33)

Total showing impairment on one 11 (33)

or more attentional/executive measure

Visuospatial function

Rey copy

Visuomotor processing speed

TMT part $\mathrm{A}$

$2(6)$

ACE, Addenbrooke's Cognitive Examination; aMCl, amnestic mild cognitive impairment; BNT, Boston Naming Test; EENT, Edinburgh Exemplar Naming Test; GFT, Graded Faces Test; GNT, Graded Naming Test; HVLT-R, Hopkins Verbal Learning Test - Revised; PAL, CANTAB Paired Associates Learning test; Rey, Rey Complex Figure Test; TMT, Trail Making Test.

\section{Neuropsychological profile of aMCI}

The applicability of Petersen's research criteria ${ }^{1}$ to clinical practice has recently been challenged on the grounds of exclusion of a significant number of patients who display episodic memory impairment of a visual nature only. ${ }^{2}$ In contrast to the results from the Cambridge Memory Clinic reported by Alladi et al, ${ }^{2}$ around half of the participants with aMCI in our study showed impairment of both verbal and visual memory, whereas all of the remaining participants with aMCI exhibited memory impairment of a verbal nature only. That is to say, we failed to uncover any case of isolated visual memory impairment.

A recent study ${ }^{7}$ supports the notion that the type of episodic memory measure used may affect whether or not impairments are detected - a point we will return to later in the discussion. However, the absence of participants showing episodic memory impairment of a solely visual nature in our sample cannot be readily explained in terms of differential test sensitivities, because nearidentical neuropsychological measures were used in previous studies (e.g. that of Alladi et $\mathrm{al}^{2}$ ) to assess visual memory function. Only a small proportion of our patients with aMCI demonstrated impairment on the visual episodic memory tasks per se. Administrative procedures might go some way to explain this observation. Specifically, our inclusion of an immediate Rey Complex Figure Test recall trial might have resulted in higher delay scores, ${ }^{27}$ thus serving to reduce the sensitivity of this measure in our aMCI group.

Findings of studies examining patients who are at risk of developing Alzheimer's disease suggest that measures of verbal episodic memory are most sensitive to changes early in the disease course, followed by measures of visual memory. ${ }^{28}$ It is therefore conceivable, taking into account our aMCI group's higher mean score on the Addenbrooke's Cognitive Examination, that our sample contained a greater number of patients who were at an earlier stage of their disease course. Longitudinal follow-up, in particular observation of annual performances on these visual episodic memory measures, will determine whether this is indeed the case.

Several studies have drawn attention to the substantial variability in MCI case definition as a function of the specific neuropsychological tests used. ${ }^{2,7}$ Consistent with this, in our study there was variability among the aMCI group as to which and how many episodic memory measures were impaired. This finding was previously demonstrated, ${ }^{2}$ and highlights the inherent difficulty in specifying the use of any single measure as a means of establishing impaired episodic memory function in aMCI. Our aMCI sample could be roughly divided into thirds in terms of numbers of participants exhibiting impairment on one, two and three or more episodic memory measures. A similar breakdown in numbers has been previously reported. ${ }^{2}$ It would appear entirely reasonable and indeed a matter of good clinical practice to seek to establish consistency in performance across a range of episodic memory measures in defining aMCI and it will be of interest to see whether this is a significant determinant of outcome.

The variability in case definition of aMCI as a function of the cognitive measures employed, coupled with the inherent difficulties in specifying the use of a single common measure in the evaluation of this condition, poses a major challenge for clinicians. Our findings suggest that employing Petersen's expanded criteria ${ }^{9}$ for MCI could conceivably lead to a patient's condition being classified as single-domain aMCI, multiple-domain aMCI or 'worried well', depending on the cognitive measures that were employed. If the MCI subdivisions prove useful in a prognostic sense, the means by which the cognitive aspects of the criteria are put into operation by clinicians will require further clarification.

Mean scores on cognitive screening measures were significantly lower for participants showing impairment on more than one episodic memory measure. This may reflect a more advanced disease course in this group. It is also possible that the single-measure impairment group will prove to be a less stable one over time, with a number of patients returning normal neuropsychological profiles when tested again at a later date. Alternatively, in cases in which participants show impairment on a single verbal memory measure only, this might have arisen secondarily to impairment in another cognitive domain, for example expressive language or attention/executive function (in which case the person's condition might be more accurately conceptualised as non-amnestic MCI). These possibilities and the prognostic implications of consistency and pervasiveness of impaired episodic memory performances remain to be examined by way of longitudinal follow-up.

Our study adds to the growing body of evidence supporting the rarity of a pure amnestic MCI syndrome, ${ }^{2,5,6}$ and demonstrates that additional impairment often goes unnoticed unless participants undergo thorough neuropsychological assessment. Among 33 patients with aMCI, only $10(30 \%)$ presented with isolated memory impairment. This figure is well within the range of previously reported rates. For example, Tabert and colleagues 
found that, following comprehensive neuropsychological assessment, only $19 \%$ of their aMCI cases were categorised as pure aMCI, ${ }^{5}$ whereas this figure reached $35 \%$ in the study by Alladi et al. ${ }^{2}$ It should be borne in mind, however, that the rate of cases with purely amnestic MCI will vary in accordance with how impairment is defined. For example, Kramer and colleagues showed that the number of cases classified as pure aMCI was considerably higher $(27 \%)$ when a cut-off of 1.5 s.d. below the mean, as opposed to 1 s.d. (resulting in a $5 \%$ rate), was used. ${ }^{6}$ It therefore remains a possibility that our less stringent definition of impairment (i.e. 1 s.d. below the mean performance of the healthy control group) might have resulted in an overestimation of the frequency of cases with non-pure aMCI. Identifying accompanying non-memory cognitive impairment none the less appears important in light of recent evidence indicating a higher risk of conversion to Alzheimer's disease in patients with aMCI who show additional areas of cognitive impairment compared with patients with pure aMCI. ${ }^{5}$

The results of our study are also consistent with evidence indicating accompanying semantic memory impairment in aMCI ${ }^{1-5}$ with just 10 patients of 33 exhibiting episodic memory impairment in isolation, and 22 of the remaining 23 displaying evidence of semantic memory compromise. This finding may reflect an increased risk of conversion to Alzheimer's disease from aMCI, although early semantic memory failure is by no means specific to the former disease, ${ }^{29,30}$ and although some studies report prognostic significance of performance on semantic memory measures, ${ }^{18,31,32}$ others have failed to do so. ${ }^{33}$ The stage at which impairments in this domain become apparent does appear to vary in accordance with the sensitivity of the measure employed. ${ }^{4}$ The intact performance of participants with aMCI on measures of lexical (letter) fluency, also previously reported, ${ }^{2,4}$ suggests that the 'initiation' aspects of semantic fluency tasks do not pose any difficulty to patients with this impairment subtype.

In view of the sound mean performances of our participants with aMCI on cognitive screening measures (MMSE score 28/30, Addenbrooke's Cognitive Examination score 88/100), it seems unlikely that consideration of such scores will be of any value in ruling out the presence of additional domains of cognitive impairment. Reliance on clinical judgement to determine the presence or absence of additional domains of subtly impaired cognition is similarly likely to prove difficult when dealing with patients with above-average premorbid IQ scores who are performing at sound levels on cognitive screens. Taken together, the above observations raise the question of whether global screening measures coupled with clinical judgement are a sufficient means of investigating $\mathrm{MCI}$, and if not, whether additional resources or an expanded skill base will be required to handle this population clinically.

Our results reveal an absence of any significant difference in performance between the aMCI and control groups on measures of visuospatial function and processing speed. In-depth longitudinal evaluation of neuropsychological performance in MCI and questionable dementia suggests that visuospatial functions tend to fail secondarily to episodic memory and category fluency performances, ${ }^{4,34}$ although some heterogeneity is known to exist. $^{35}$ It is therefore once again possible that our failure to demonstrate group differences on a visuospatial copying task reflects an earlier disease stage of our aMCI sample. Alternatively, it is conceivable that the varied and somewhat subjective scoring methods for the Rey Complex Figure Test copy task across different studies might be responsible for this finding. Crosssectional findings pertaining to visuomotor processing speed in MCI vary, with some studies reporting significant differences between MCI and control groups ${ }^{1,36,37}$ and others, like ourselves, failing to do so. ${ }^{38-40}$ The disparity in findings may simply reflect the heterogeneity of aMCI or alternatively the disease stage. Group differences in processing speed might be more likely to exist where samples contain significant numbers of patients in the preclinical stages of a subcortical dementia of a cerebrovascular nature. For example, there is some evidence to suggest a disproportionately strong association between perceptual speed and parkinsonian signs in MCI. ${ }^{41}$

\section{Limitations}

Several study limitations should be noted. The significantly higher mean age of our patient group opens up the possibility that some of their performance deficits were explicable in terms of agerelated cognitive decline. Ideally, control for age should have been better. However, aMCI group participants were identified on the basis of their performance on age-standardised tests; therefore, the discrepancy would not have influenced patient group membership. Longitudinal follow-up of these patients will help to clarify the relevance of this difference. Furthermore, our aMCI sample was characterised by a high average level of estimated premorbid general intellectual function, which introduces problems of applicability. Similar issues were present in a recent comparable study, ${ }^{2}$ although other socio-demographic characteristics (i.e. gender, ethnicity, education and occupation) were not reported, preventing further comparison between that and our study. There may therefore be a need to replicate these findings employing greater numbers of age- and IQ-matched healthy controls and aMCI patients with average premorbid IQs, together with other socio-demographic markers more closely resembling the population mean.

\section{Implications}

Patients with MCI make up a significant number of referrals to older adult memory assessment services, with the most common referral subtype in our sample being that of aMCI, followed by equal numbers of non-amnestic MCI and worried well. Relatively few people with aMCI exhibit episodic memory compromise in isolation and fewer still show a visual but not verbal episodic memory deficit. Both the concept and criteria for MCI therefore appear to be relevant and indeed necessary adjuncts to clinical practice.

Our findings highlight the inherent difficulties of specifying a single measure in the assessment of memory and other cognitive functions in MCI, while at the same time emphasising the need for clarification of the means by which MCI criteria can be put into operation clinically. Initial attempts at better defining neuropsychological aspects of the aMCI criteria have been made, ${ }^{1}$ but their application in a clinical sense remains inconsistent and their poor definition has not gone unnoticed. ${ }^{42}$ The existence of a number of neuropsychological measures of well-documented sensitivity in aMCI and the strikingly similar mean performances of different clinic aMCI groups on such measures suggest that this need not be the case. Although the importance of exercising clinical judgement in arriving at a diagnosis of MCI cannot be ignored, it would none the less seem inevitable that further definition of the neuropsychological aspects of MCI criteria will be needed to facilitate identification of the subtypes of impairment and to further our understanding of their respective prognoses.

\section{Acknowledgements}

This study was financially supported by the Gordon Small Charitable Trust and the European Commission Network of Excellence Diagnostic Molecular Imaging (FP6-LIFESCl- 
HEALTH Project Reference 512146). We thank all the participants and our clinical colleagues for referring their patients.

Jane A. Lonie, MSc, University of Edinburgh Division of Psychiatry, Royal Edinburgh Hospital, Edinburgh; Lucie L. Herrmann, MA, University of Oxford Department of Psychiatry, Warneford Hospital, Oxford; Claire L. Donaghey, MSc, University of Edinburgh Division of Psychiatry, Royal Edinburgh Hospital, Edinburgh; Klaus P. Ebmeier, FRCPsych, University of Edinburgh Division of Psychiatry, Royal Edinburgh Hospital, Edinburgh, and University of Oxford Department of Psychiatry, Warneford Hospital, Oxford, UK

Correspondence: Professor K. P. Ebmeier, Old Age Psychiatry Group, Department of Psychiatry, University of Oxford, Warneford Hospital, Oxford Ox3 7JX, UK. E-mail: klaus.ebmeier@psych.ox.ac.uk

First received 16 January 2007, final revision 11 April 2007, accepted 16 May 2007

\section{References}

1 Grundman M, Petersen RC, Ferris SH, Thomas RG, Aisen PS, Bennett DA Foster NL, Jack CR, Jr, Galasko DR, Doody R, Kaye J, Sano M, Mohs R, Gauthier S, Kim HT, Jin S, Schultz AN, Schafer K, Mulnard R, van Dyck $\mathrm{CH}_{\text {, }}$ Mintzer J, Zamrini EY, Cahn-Weiner D, Thal LJ. Mild cognitive impairment can be distinguished from Alzheimer disease and normal aging for clinical trials. Arch Neurol 2004; 61: 59-66.

2 Alladi S, Arnold R, Mitchell J, Nestor PJ, Hodges JR. Mild cognitive impairment: applicability of research criteria in a memory clinic and characterization of cognitive profile. Psychol Med 2006; 36: 507-15.

3 Ribeiro F, de Mendonça A, Guerreiro M. Mild cognitive impairment: deficits in cognitive domains other than memory. Dement Geriatr Cogn Dis 2006; 21: 284-90.

4 Hodges JR, Erzinclioglu S, Patterson K. Evolution of cognitive deficits and conversion to dementia in patients with mild cognitive impairment: a verylong-term follow-up study. Dement Geriatr Cogn Dis 2006; 21: 380-91.

5 Tabert MH, Manly JJ, Liu X, Pelton GH, Rosenblum S, Jacobs M, Zamora D, Goodkind M, Bell K, Stern Y, Devanand DP. Neuropsychological prediction of conversion to Alzheimer disease in patients with mild cognitive impairment. Arch Gen Psychiatry 2006; 63: 916-24.

6 Kramer JH, Nelson A, Johnson JK, Yaffea K, Glenna S, Rosena HJ, Millera BL. Multiple cognitive deficits in amnestic mild cognitive impairment. Dement Geriatr Cogn Dis 2006; 22: 306-11.

7 Loewenstein DA, Acevedo A, Ownby R, Agron J, Barker WW, Isaacson R, Strauman S, Duara R. Using different memory cutoffs to assess mild cognitive impairment. Am J Geriatr Psychiatry 2006; 14: 911-19.

8 Petersen RC, Stevens JC, Ganguli M, Tangalos EG, Cummings JL, DeKosky ST. Practice parameter: early detection of dementia: mild cognitive impairment (an evidence-based review). Report of the Quality Standards Subcommittee of the American Academy of Neurology. Neurology 2001; 56: 1133-42.

9 Petersen RC. Mild cognitive impairment as a diagnostic entity. J Intern Med 2004; 256: 183-94.

10 Petersen RC, Morris JC. Mild cognitive impairment as a clinical entity and treatment target. Arch Neurol 2005; 62: 1160-3.

11 Folstein MF, Folstein SE, McHugh PR. 'Mini-mental state': a practical method for grading the cognitive state of patients for the clinician. J Psychiatr Res 1975; 12: 189-98.

12 Mathuranath PS, Nestor PJ, Berrios GE, Rakowicz W, Hodges JR. A brie cognitive test battery to differentiate Alzheimer's disease and frontotemporal dementia. Neurology 2000; 55: 1613-20.

13 Morris JC. The Clinical Dementia Rating (CDR): current version and scoring rules. Neurology 1993; 43: 2412-14.

14 American Psychiatric Association. Diagnostic and Statistical Manual of Mental Disorders (4th edn) (DSM-IV). APA, 1994.

15 Yesavage JA, Brink TL, Rose TL, Lum O, Huang V, Adey MB, Leirer VO. Development and validation of a geriatric depression screening scale: a preliminary report. J Psychiatr Res 1982; 17: 37-49.

16 Nelson H, Willison J. National Adult Reading Test (NART). nferNelson, 1991.

17 Brandt J. The Hopkins Verbal Learning Test: development of a new memory test with 6 equivalent forms. Clin Neuropsychol 1991; 5: 125-42.

18 Blackwell AD, Sahakian BJ, Vesey R, Semple JM, Robbins TW, Hodges JR. Detecting dementia: novel neuropsychological markers of preclinical Alzheimer's disease. Dement Geriatr Cogn Dis 2004; 17: 42-8.

19 Swainson R, Hodges JR, Galton CJ, Semple J, Michaele A, Dunna BD, Iddonf JL, Robbins TW, Sahakian BJ. Early detection and differential diagnosis of Alzheimer's disease and depression with neuropsychological tasks. Dement Geriatr Cogn Dis 2001; 12: 265-80.

20 Rey A. Psychological examination of a case of post-traumatic encephalopathy. Arch Psychol 1941; 28: 286-340.

21 McKenna P, Warrington EK. Graded Naming Test. nferNelson, 1983.

22 Thompson SA, Graham KS, Patterson K, Sahakian BJ, Hodges JR. Is knowledge of famous people disproportionately impaired in patients with early and questionable Alzheimer's disease? Neuropsychology 2002; 16: 344-58

23 Kaplan E, Goodglass H, Weintraub S. Boston Naming Test. Lea \& Febiger, 1983.

24 Reitan RM. Halstead-Reitan Neuropsychological Test Battery. Reitan Neuropsychology Laboratory Press, 1985.

25 Lehrner J, Gufler R, Guttmann G, Maly J, Gleiss A, Auff E, Dal-Bianco P. Annual conversion to Alzheimer disease among patients with memory complaints attending an outpatient memory clinic: the influence of amnestic mild cognitive impairment and the predictive value of neuropsychological testing. Wien Klin Wochenschr 2005; 117: 629-35.

26 Lopez OL, Jagust WJ, DeKosky ST, Becker JT, Fitzpatrick A, Dulberg C, Breitner J, Lyketsos C, Jones B, Kawas C, Carlson M, Kuller LH. Prevalence and classification of mild cognitive impairment in the Cardiovascular Health Study Cognition Study: Part 1. Arch Neurol 2003; 60: 1385-9.

27 Lezak MD, Howieson DB, Loring DW, Hannay HJ, Fischer JS. Neuropsychological Assessment. Oxford University Press, 2004.

28 Collie A, Maruff P. The neuropsychology of preclinical Alzheimer's disease and mild cognitive impairment. Neurosci Biobehav Rev 2000; 24: 365-74.

29 Clague F, Dudas RB, Thompson SA, Graham KS, Hodges JR. Multidimensional measures of person knowledge and spatial associative learning: can these be applied to the differentiation of Alzheimer's disease from frontotemporal and vascular dementia? Neuropsychologia 2005; 43: 1338-50.

30 Graham NL, Emery T, Hodges JR. Distinctive cognitive profiles in Alzheimer's disease and subcortical vascular dementia. J Neurol Neurosurg Psychiatr 2004; 75: 61-71

31 Artero S, Tierney MC, Touchon J, Ritchie K. Prediction of transition from cognitive impairment to senile dementia: a prospective, longitudinal study. Acta Psychiatr Scand 2003; 107: 390-3.

32 Estevez-Gonzàlez A, Garcia-Sànchez C, Boltes A, Otermín P, Pascual-Sedano $B$, Gironell A, Kulisevsky J. Semantic knowledge of famous people in mild cognitive impairment and progression to Alzheimer's disease. Dement Geriatr Cogn Dis 2004; 17: 188-95.

33 Beatty WW, Salmon DP, Troster Al, Tivis RD. Do primary and supplementary measures of semantic memory predict cognitive decline by patients with Alzheimer's disease? Neuropsychol Dev Cogn B Aging Neuropsychol Cogn 2002; 9: 1-10

34 Perry RJ, Hodges JR. Fate of patients with questionable (very mild) Alzheimer's disease: Iongitudinal profiles of individual subjects' decline. Dement Geriatr Cogn Dis 2000; 11: 342-9.

35 Caine D, Hodges JR. Heterogeneity of semantic and visuospatial deficits in early Alzheimer's disease. Neuropsychology 2001; 15: 155-64.

36 Arnaiz E, Almkvist O, Ivnik RJ, Tangalos EG, Wahlund LO, Winblad B, Petersen RC. Mild cognitive impairment: a cross-national comparison. J Neurol Neurosurg Psychiatr 2004; 75: 1275-80.

37 Nordlund A, Rolstad S, Hellstrom P, Sjogren M, Hansen S, Wallin A. The Goteborg $\mathrm{MCl}$ study: mild cognitive impairment is a heterogeneous condition. J Neurol Neurosurg Psychiatr 2005; 76: 1485-90.

38 Albert MS, Moss MB, Tanzi R, Jones K. Preclinical prediction of AD using neuropsychological tests. J Int Neuropsychol Soc 2001; 7: 631-9.

39 Crowell TA, Luis CA, Rodney DV, Schinka JA, Mullan M. Memory patterns and executive functioning in mild cognitive impairment and Alzheimer's disease. Neuropsychol Dev Cogn B Aging Neuropsychol Cogn 2002; 9: 288-97.

40 Fox NC, Warrington EK, Seiffer AL, Agnew SK, Rossor MN. Presymptomatic cognitive deficits in individuals at risk of familial Alzheimer's disease. A longitudinal prospective study. Brain 1998; 121: 1631-9.

41 Boyle PA, Wilson RS, Aggarwal NT, Arvanitakis Z, Kelly J, Bienias JL, Bennett DA. Parkinsonian signs in subjects with mild cognitive impairment. Neurology 2005; 65: 1901-6.

42 Portet F, Ousset PJ, Visser PJ, Frisoni GB, Nobili J, Scheltens Ph, Vellas B Touchon J. Mild cognitive impairment $(\mathrm{MCl})$ in medical practice: a critical review of the concept and new diagnostic procedure. Report of the $\mathrm{MCl}$ Working Group of the European Consortium on Alzheimer's Disease. J Neurol Neurosurg Psychiatr 2006; 77: 714-18. 